\title{
Joint Genome Institute's Automation Approach and History
}

\author{
June 27, 2006 \\ Simon Roberts \\ (Production Instrumentation Supervisor)
}

This work was performed under the auspices of the US Department of Energy's Office of Science, Biological and Environmental Research Program, and by the University of California, Lawrence Livermore National Laboratory under Contract No. W-7405-Eng-48, Lawrence Berkeley National Laboratory under contract No. DE-AC02-05CH11231 and Los Alamos National Laboratory under contract No. DE-AC02-06NA25396 


\section{Agenda}

- Brief overview of the how the Joint Genome Institute came into existence.

- Overview of DNA sequencing production line at the JGI.

- How our throughput has increased since 1999 to become a high through-put sequencing facility.

- Some instrumentation improvement highlights along the way and how they are used.

- Review our approach to successful selection \& implementation of new instruments to meet our needs.

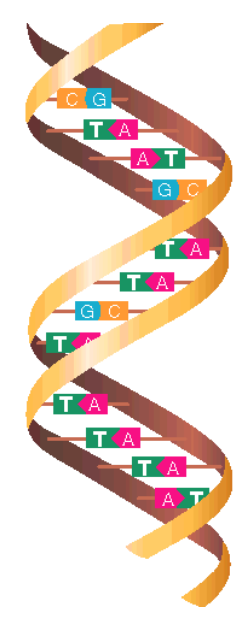




\section{JIO DOE JGI \\ Production Genomics Facility}

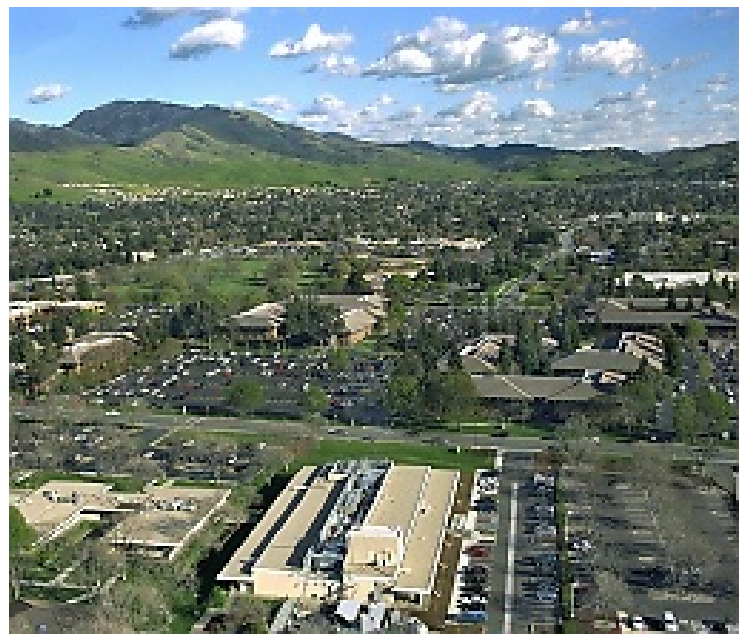

Opened in 1999

240 UC Employees

$60,000 \mathrm{sf}$

$\sim$ \$66M Annual Budget

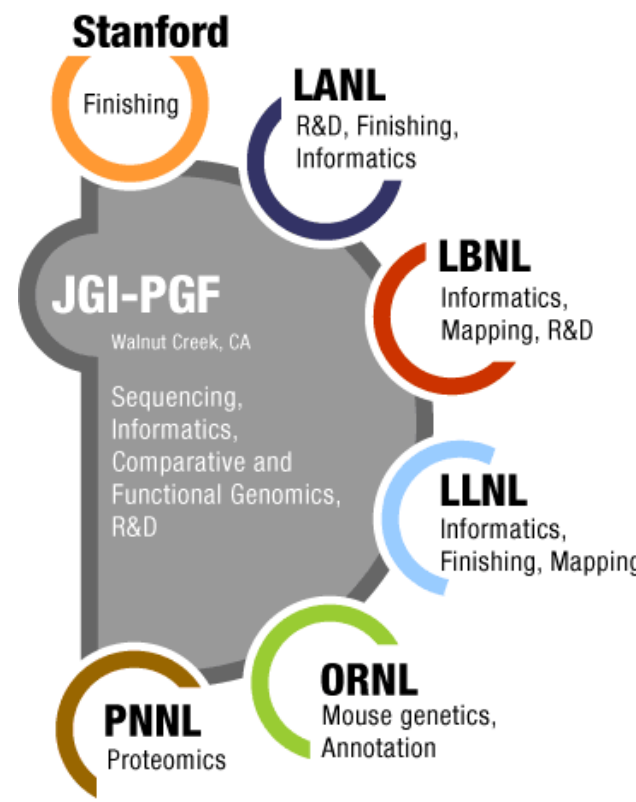

The DOE Joint Genome Institute (JGI) is a "virtual institute" that integrates the sequencing and

Mission:
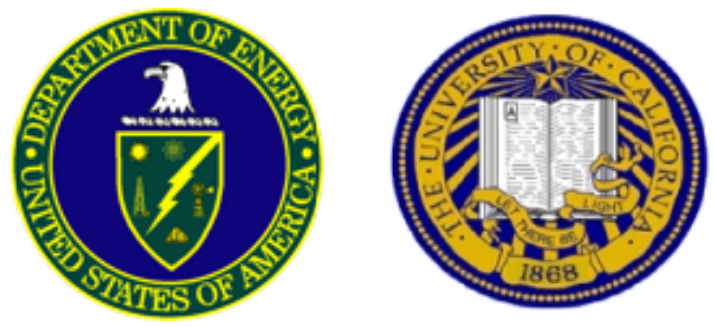
institutions:

DOE JGI collaborates with DOE national laboratories and community users, to advance genome science in support of the DOE missions of clean bio-energy, carbon cycling, and bioremediation. 


\section{Important Dates in DOE Genomics}

- 1986 DOE announces Human Genome Initiative.

With $\$ 5.3$ million, pilot projects begin at DOE national laboratories to develop critical resources and technologies.

- 1990 DOE \& NIH present their joint HGP plan to Congress. The 15-year project formally begins.

- 1997 DOE creates the JGI uniting activities at DOE human genome centers.

- 1999 JGI opens the Production Genomics Facility (PGF) in Walnut Creek, staff from LLNL \& LBNL.

- 2000 HGP leaders \& President Clinton announce the completion of a "working draft..the first great technological triumph of the 21st century."
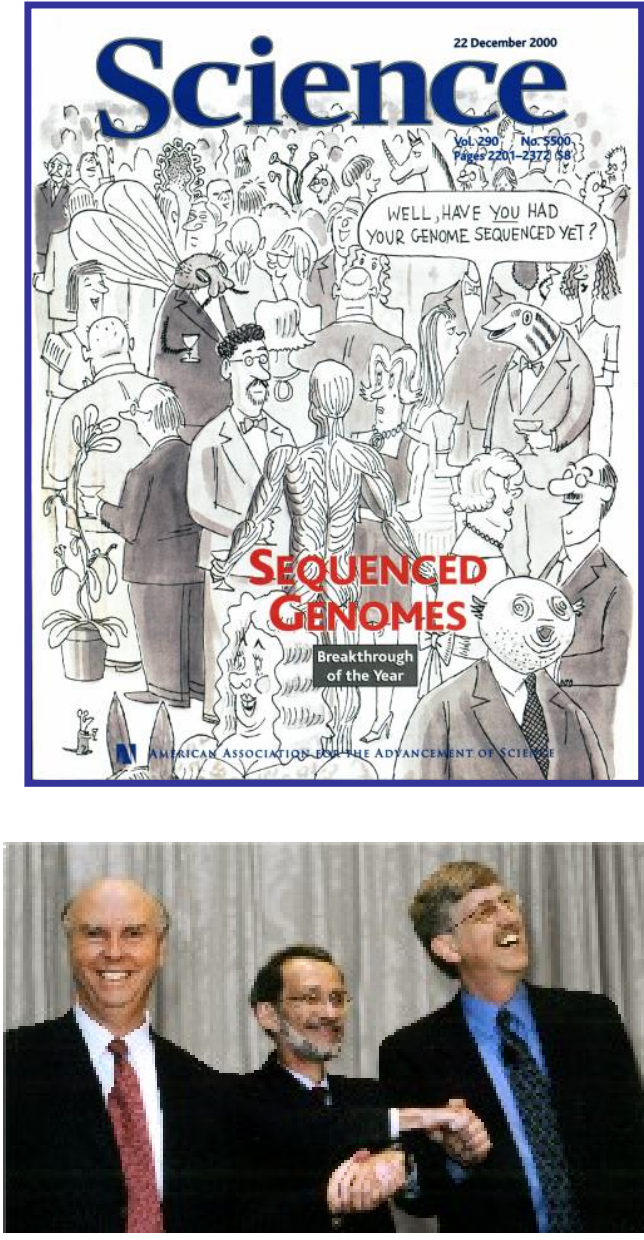

- 2003 HGP completed and published. 


\section{What IS a Genome???}

A GENOME is all of a living thing's genetic material.

The genetic material is DNA (DeoxyriboNucleic Acid) DNA, a double helical molecule, is made up of four nucleotide "letters":

A--<smiles>Nc1cccc2cccnc12</smiles><smiles>Nc1cc2c(cc1N)C(=O)NCC2</smiles>

T--

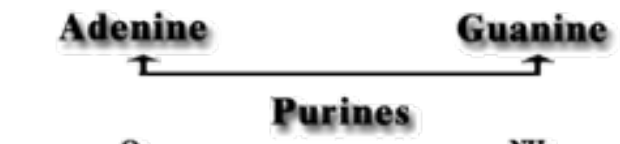<smiles>CC1CC=CC(=O)C1=O</smiles><smiles>CC1=CC(=O)CC=N1</smiles>
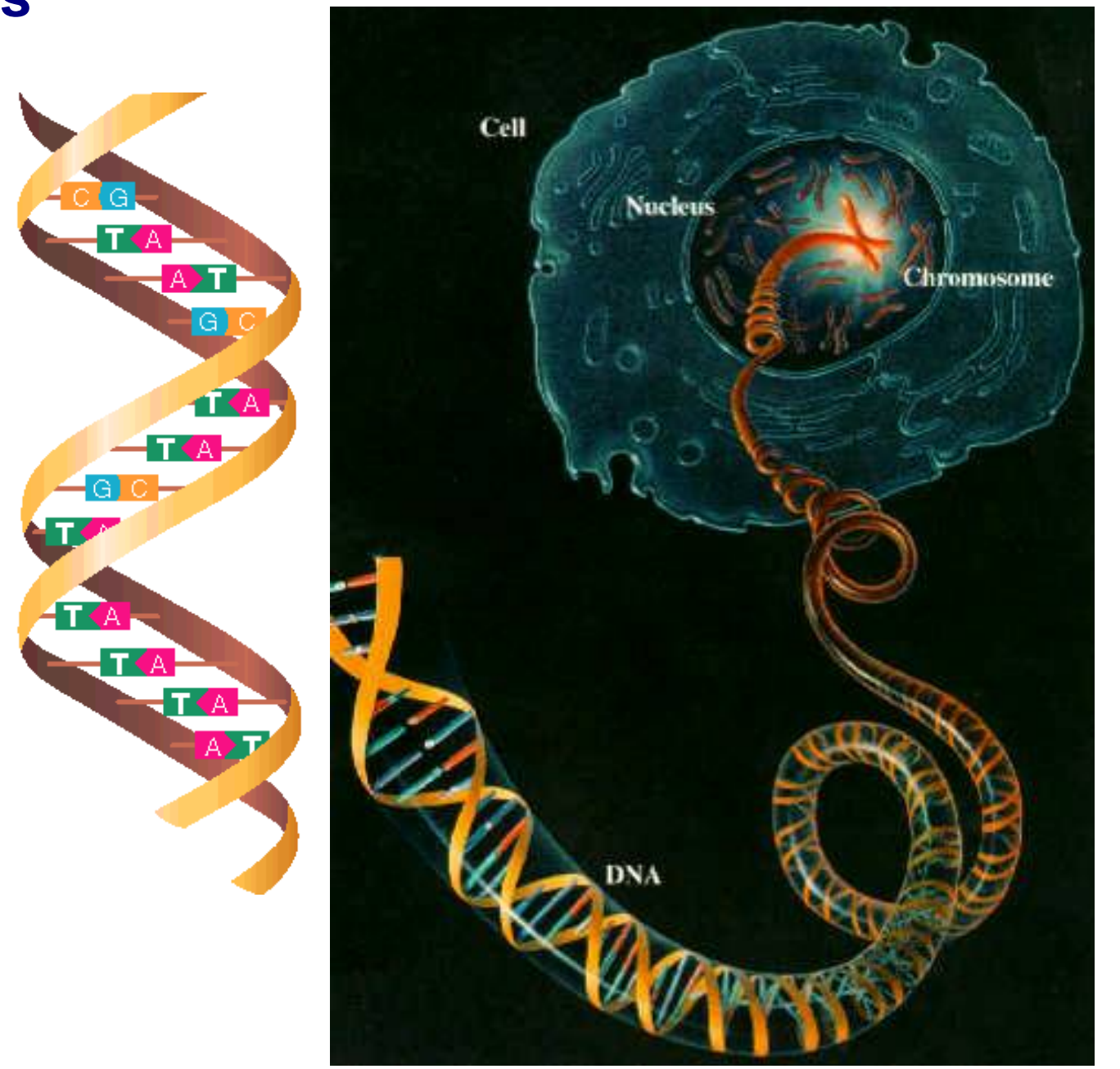

Pyrimidines

C--

G--

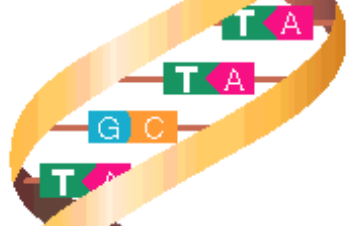

$\rightarrow$

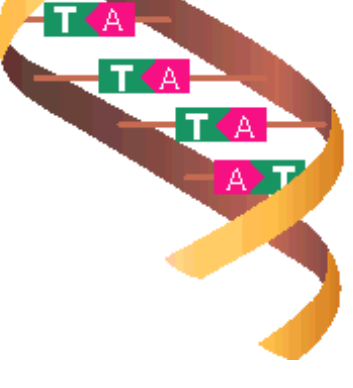

Thymine

Cytosine 


\section{JGI Production Mission}

\section{"Produce high quality cost efficient 'assemble-able' sequence in a safe environment."}

FY 2006 Goals

- 52 million lanes $=35$ billion bases 


\section{How Sequencing is Done}

\section{Production Line Overview}

- Library Creation

- Shearing the DNA (Genomic Solutions Hydroshear)

- Insertion of Fragments into Plasmid (Ligation)

- Transformation (Electroporation)

- Subcloning the Sheared Fragment (Plating)

- Colony Picking (Genetix QPix)

- Production Sequencing

- Lysing the Cell (Matrix PlateMate)

- Rolling Circle Amplification (MultiDrop Micro)

- Sequencing Chemistry (CyBio Vario)

- Post Sequencing Reaction Cleanup (BioMek FX)

- Capillary Sequencing (ABI 3730 \& MB 4500)

- Assembly \& QA

- Assembly (Phrap, JAZZ)

- Quality Assessment (Phred Q20)
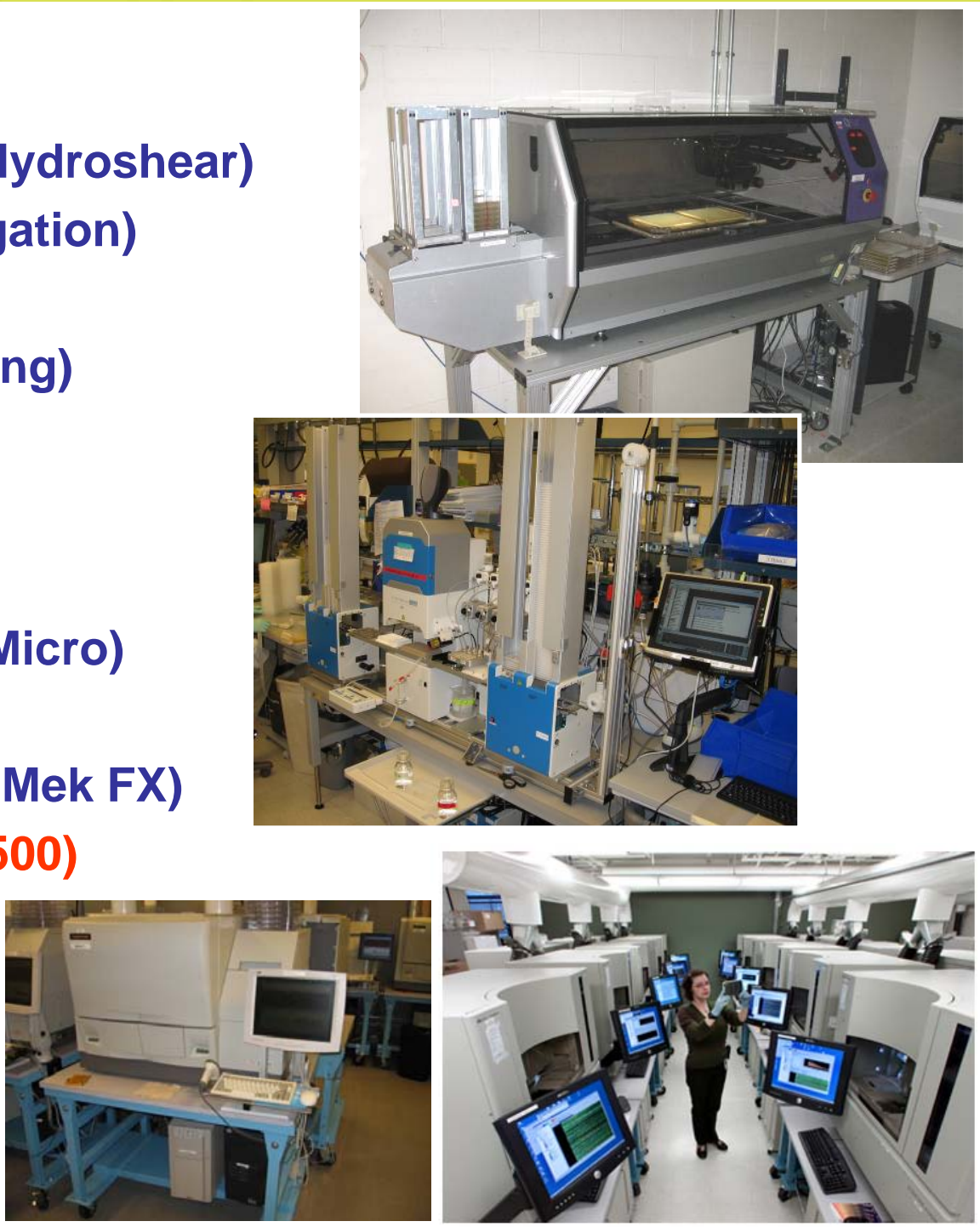


\section{DOE JG]}

\section{Production Sequencing Efficiencies}

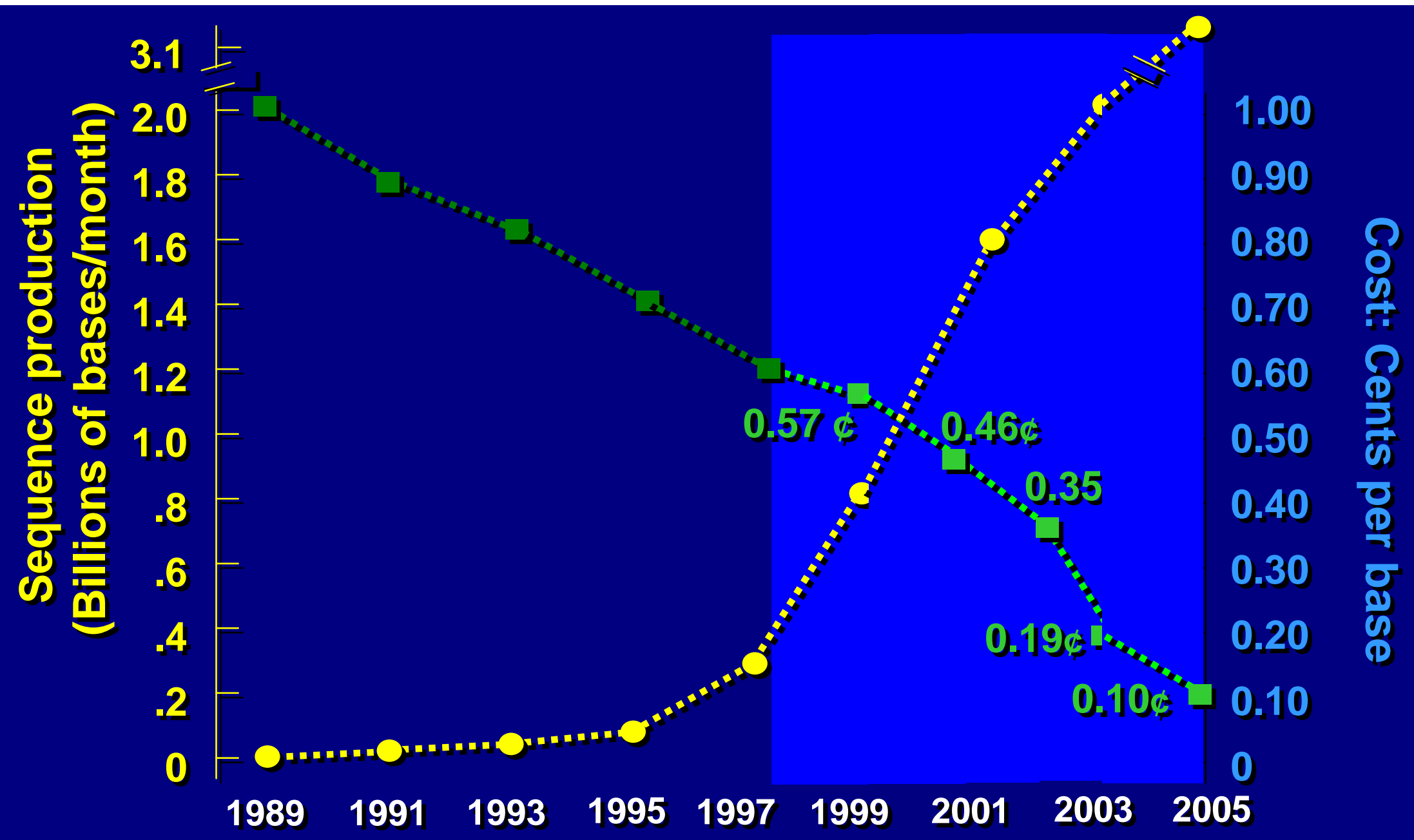




\section{"Moore's Law" of DNA Sequencing}

- April 2002:

- January 2004:

- July 2004:

- March 2005:
$1 \mathrm{~Gb} /$ month

$2 \mathrm{~Gb} /$ month

$2.5 \mathrm{~Gb} / \mathrm{month}$

3.1 Gb/month

(equivalent to 1 human genome/month)

Total (3/99-4/06) $114 \mathrm{~Gb}$

(equivalent of sequencing 38 human genomes)

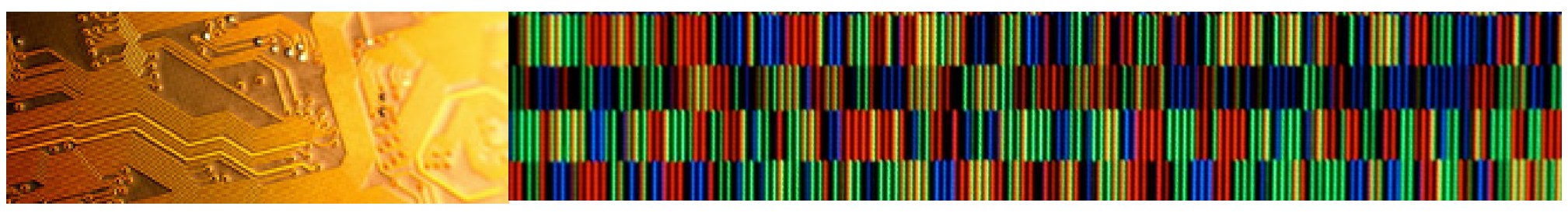

Main Production Metrics; Read Length \& Pass Rate 


\section{JG New Technologies Fuel Growth in \\ Capacity and Reduce Costs}

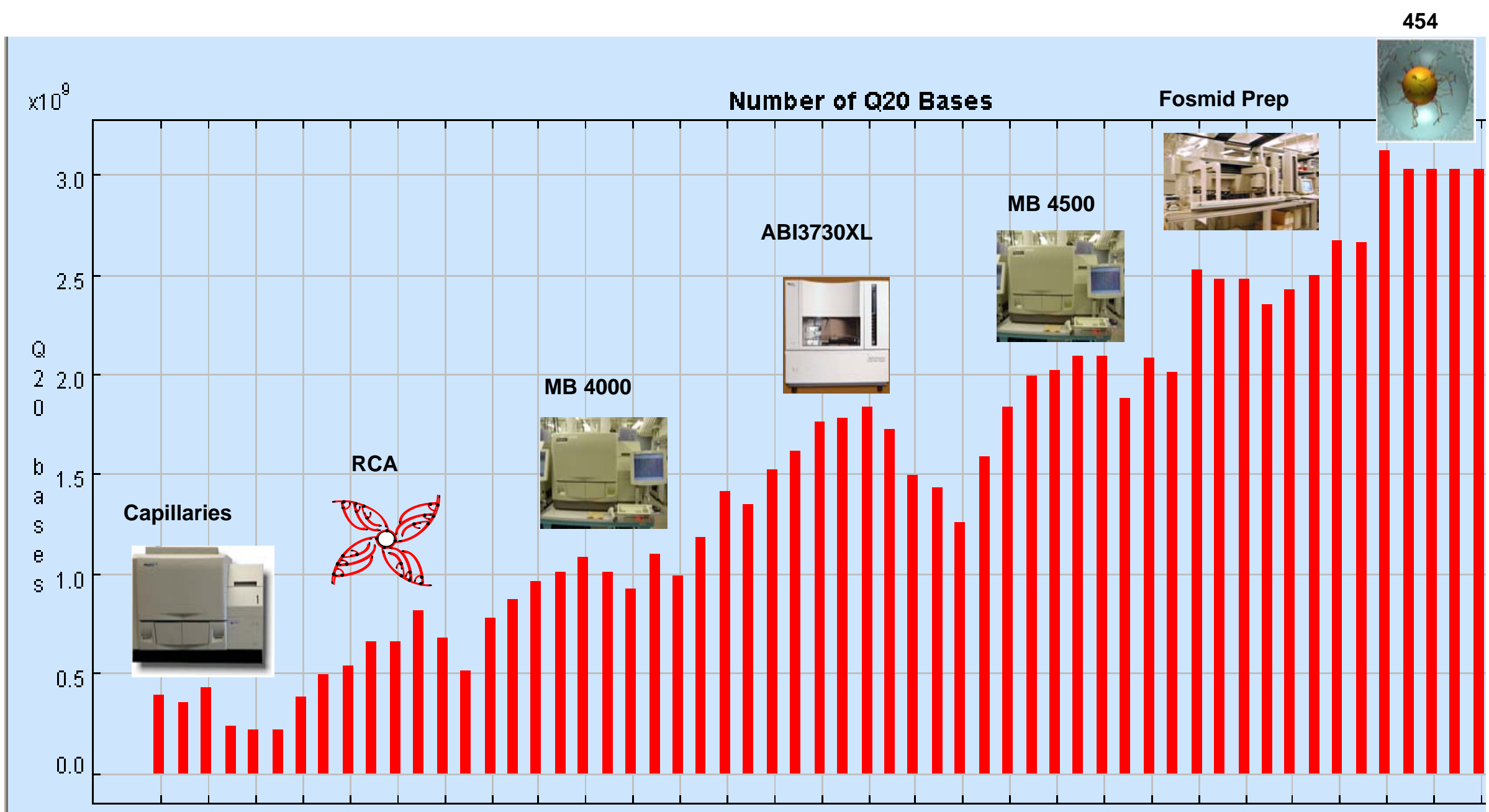

Now1.JanMar May Jul Sep Now2JanMar May Jul Sep Now03.JanMar May Jul Sep Now04JanMar May Jul Sep Now05JanMar May Ju 


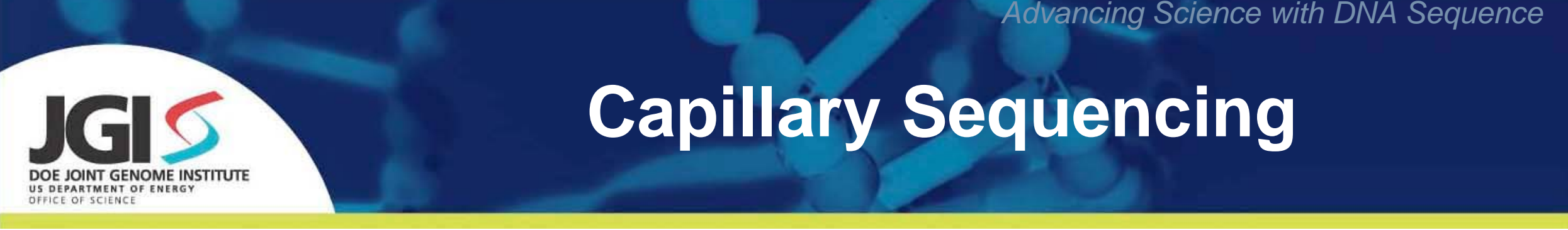

\section{MegaBace}

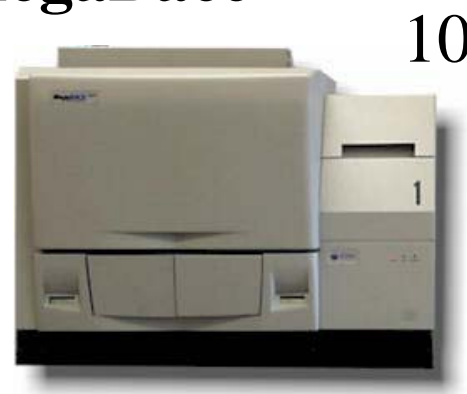

Qty 10; 2000 to Qty 84; 2002

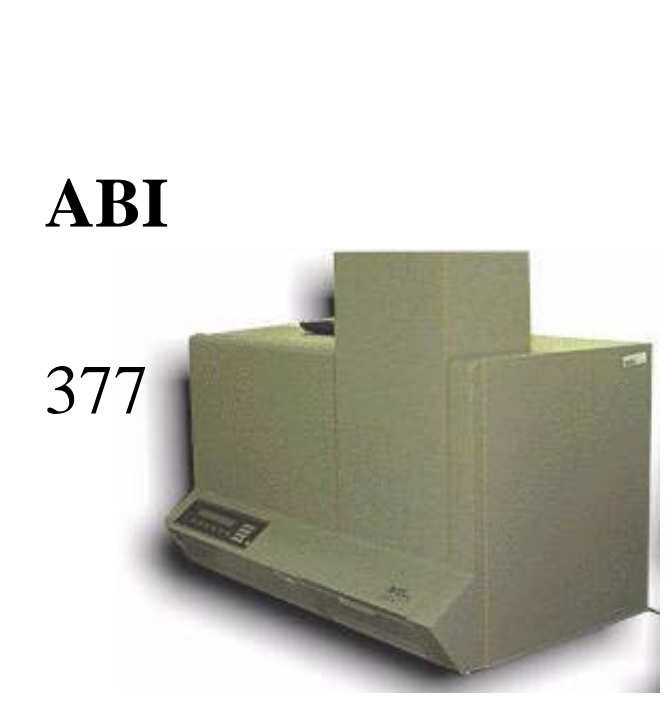

Qty 28; 1997

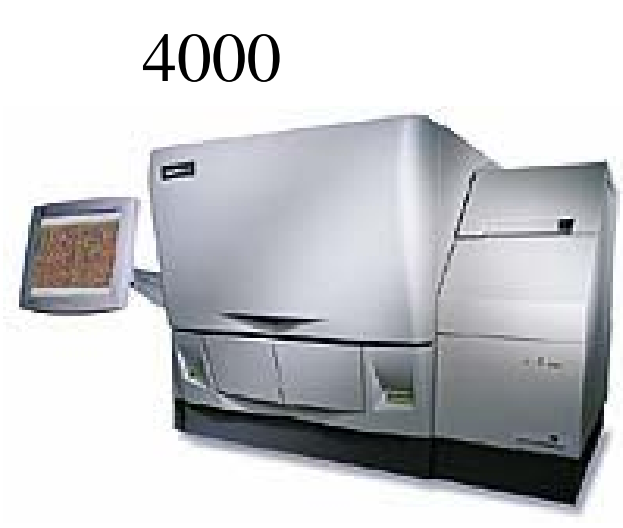

Qty 21; 2002 to Qty 36, 2003

7 days a week operation

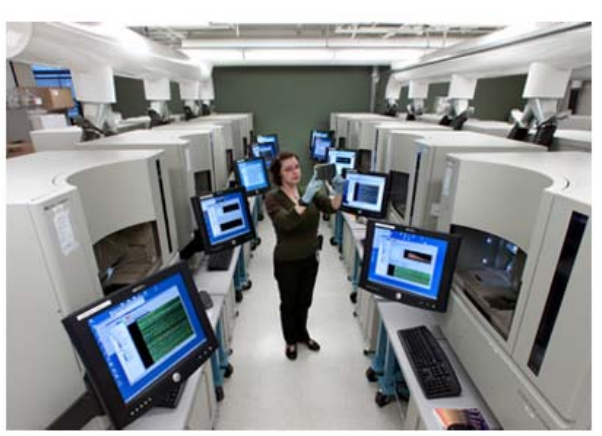

Qty 5; 2001 Qty 35; 2002 Qty 55; 2003

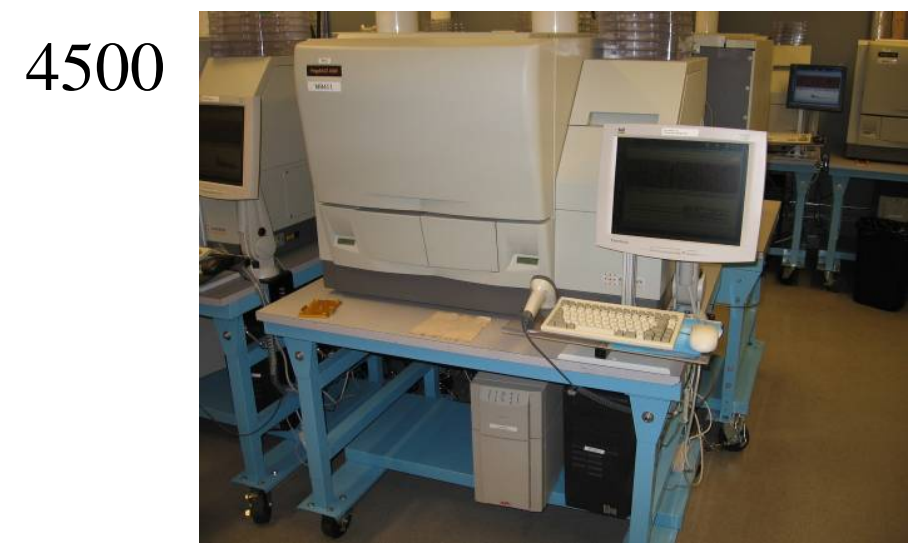

Qty 36, upgraded 2004 400 plates/day

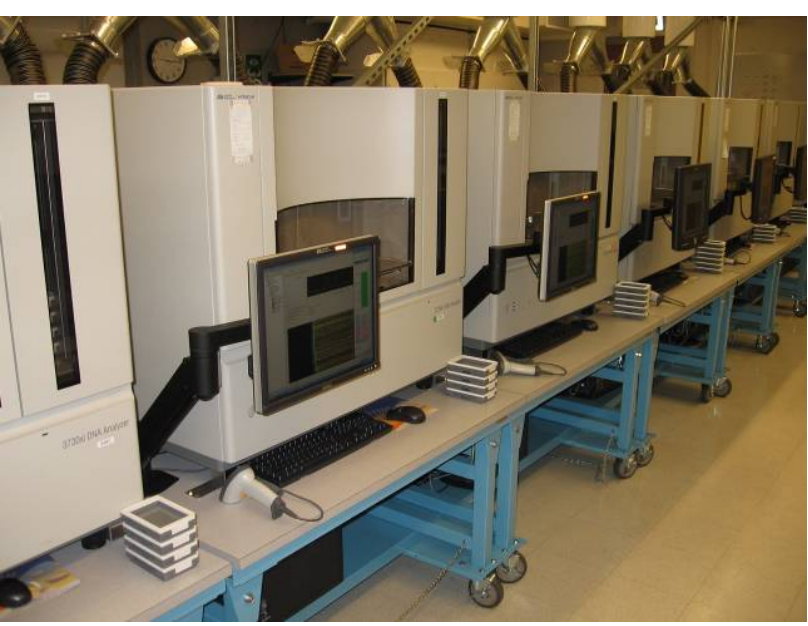

Qty 70; 2004 


\section{Jyr Throughput Increases \\ Correlated with \# Sequencers}

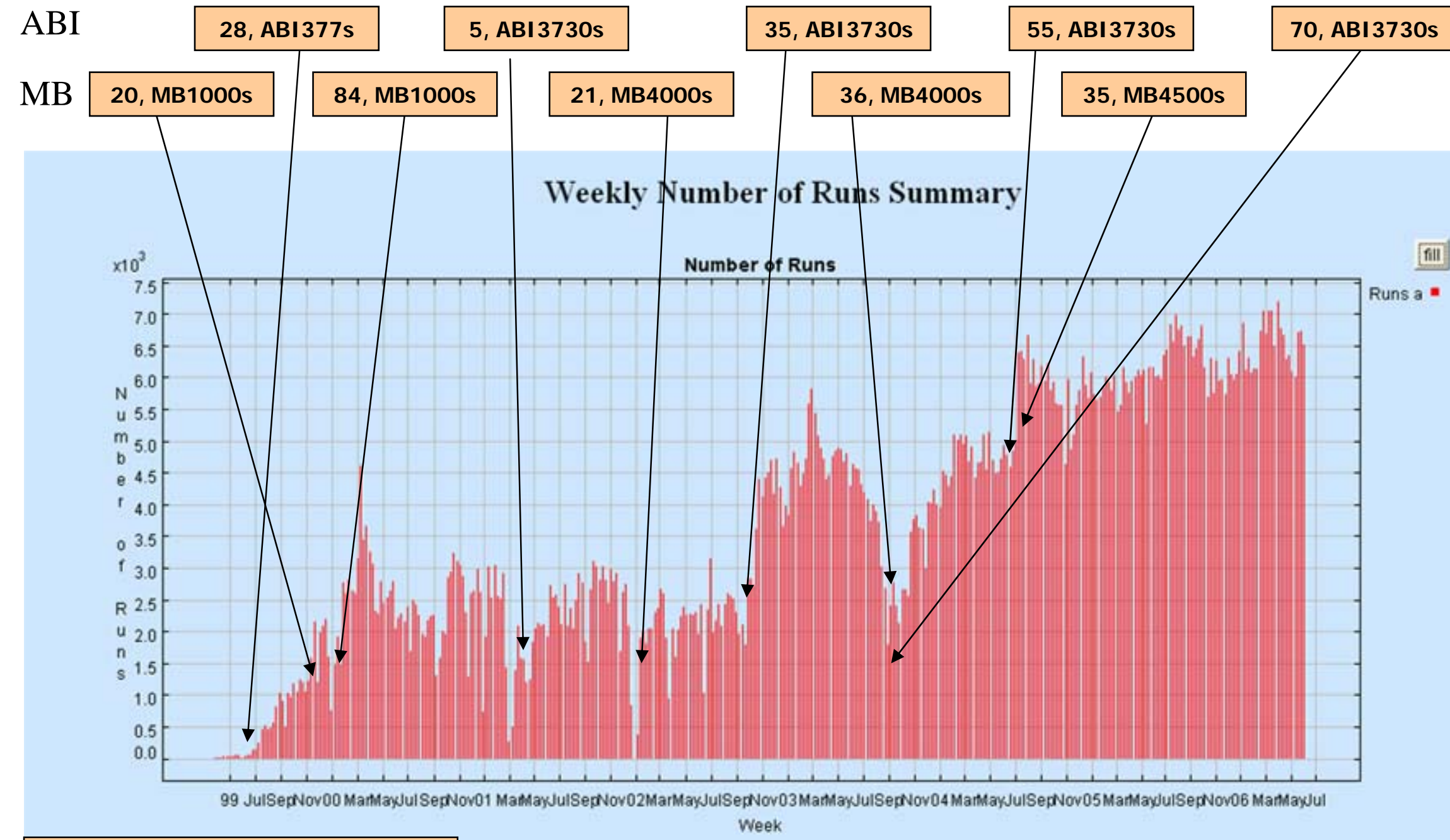

TOTAL, Sequencer Type 


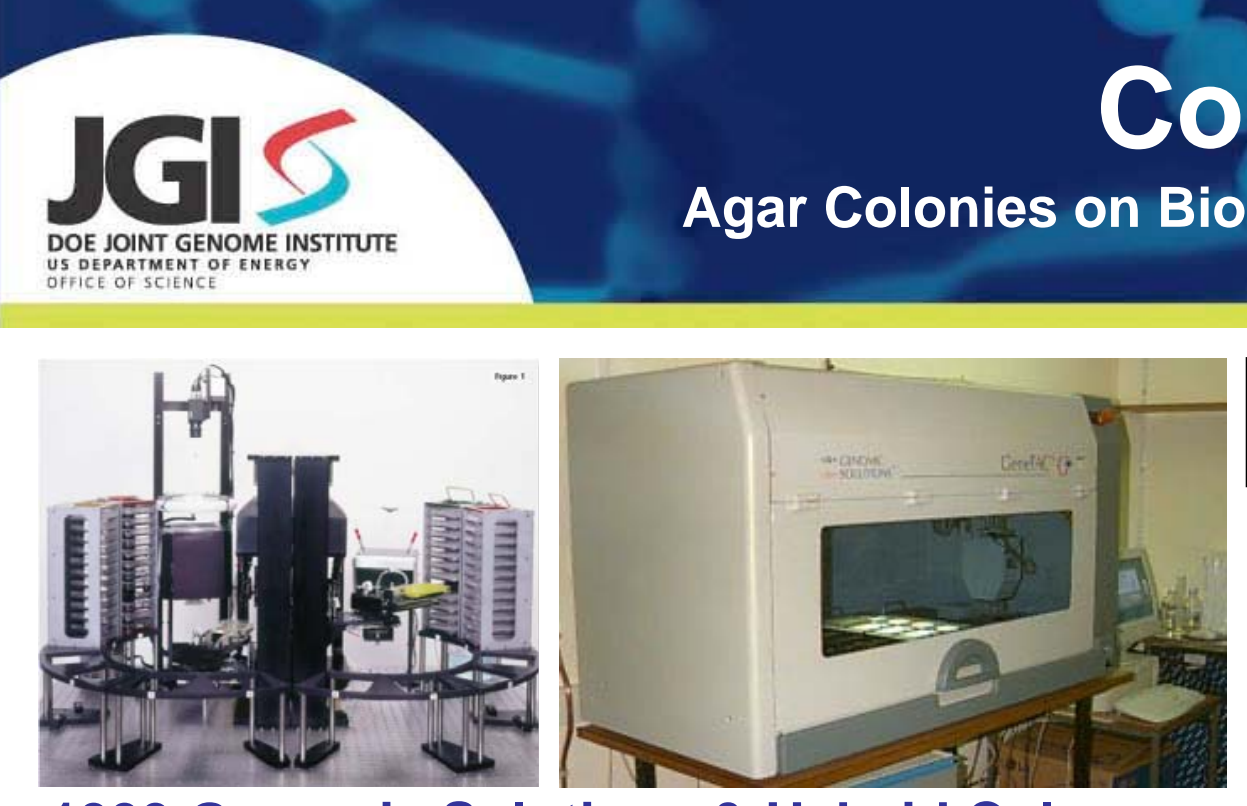

1999 Genomic Solutions \& Hybaid Colony Pickers

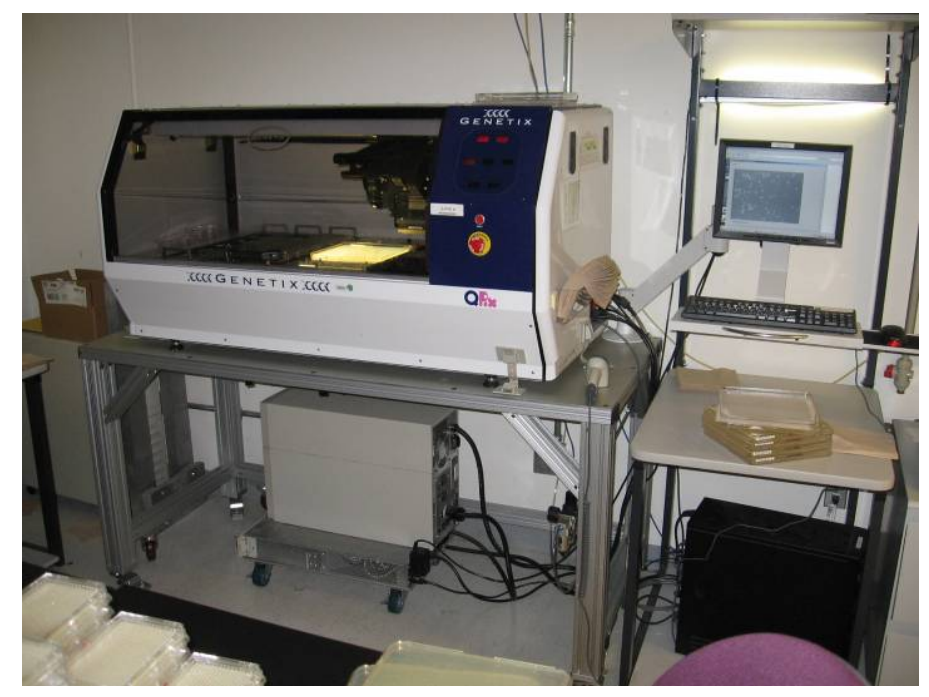

QPix2 (Qty 1; 2000, Qty 3; 2001, Qty4; 2002)

\section{OLD Throughput Trays/day}

96 pin picking head

Throughput (2 shifts $=16 \mathrm{hrs}$ )

-115 Bioassay Trays to produce 300 (384 well) destination plates/day based on $\sim 1000$ colonies/tray

\section{NEW}

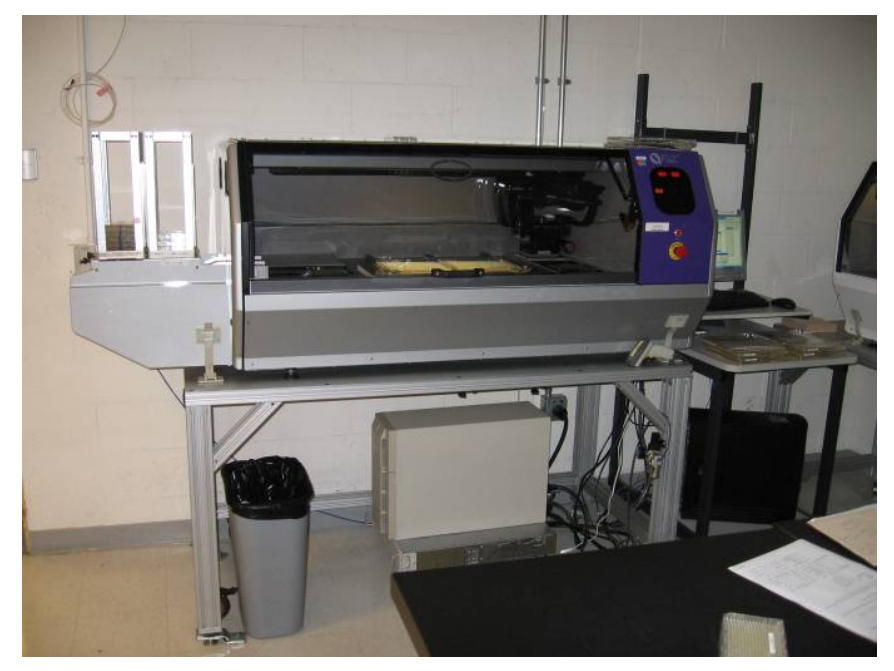

2 BioAssays \& Stacked Dest Plates 


\section{Sequence Chemistry}

Aliquot RCA Product to Daughter Plates

\& Dispense FWD \& REV Chemistry

Hydra-Twister

System

Qty 2; 2001

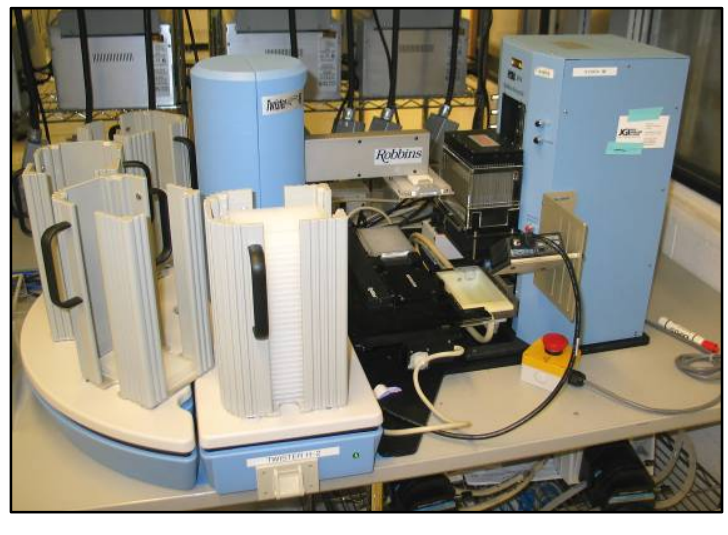

21 source to 42

destination $\sim 1 \mathrm{hr} / \mathrm{batch}$
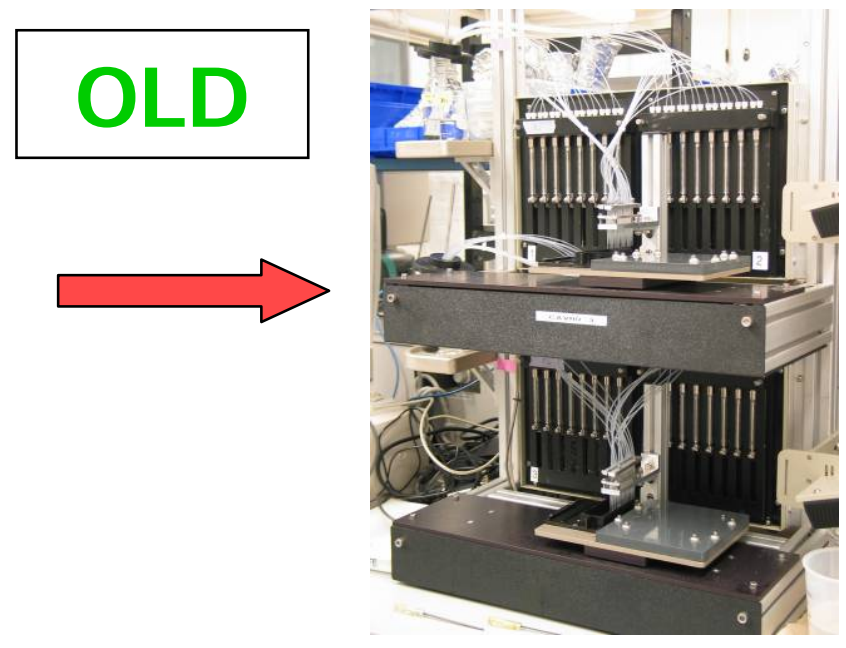

Cavro Syringe Pump Dispenser System

42 plates $\sim 40 \mathrm{~min} / \mathrm{batch}$

Typical Aliquot 1.5uL RCA Product into two destination plates followed by $3.5 \mathrm{uL}$ Sequencing Chemistry Cocktail Dispense Throughput (2 shifts $=16 \mathrm{hrs}$ )

6 batches, 42 source plates $=84$ destination plates 500+ plates/day

CyBi-Well Vario Integrated System Qty 2; 2006

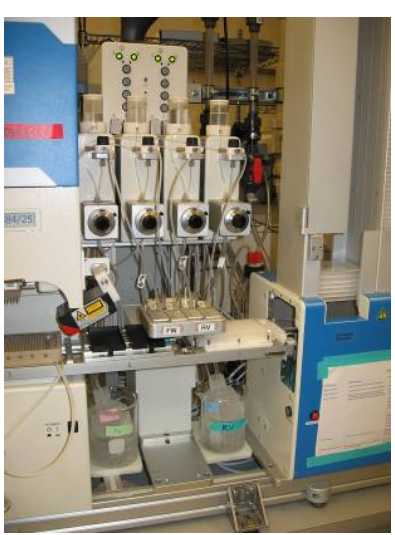

2.5hr/batch

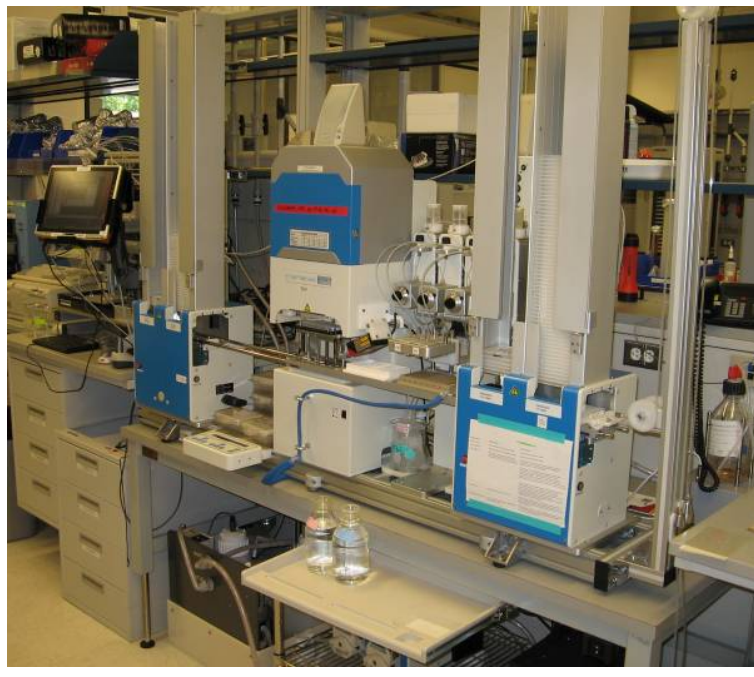




\section{Why Automate?}

- In high throughput environment;

- automation does not necessarily increase productivity of workers,

- does increase repeatability

- increase equipment reliability

- frees up operator to perform other tasks

- reduce risk to operator of ergonomic issues

- "Islands of Automation"

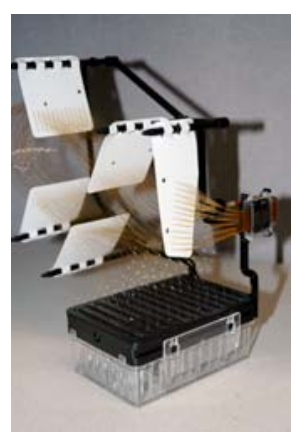

- modular instruments with stacks of micro-titer plates transported \& loaded by operators

- Semi-Automated Approach

- minimizes cost and maximizes flexibility

- compared to fully robotic systems

- presents a unique set of challenging issues when moving large volumes of plates 


\section{Why Automate?}

- Need to automate driven by;

- Lowering Reagent Volumes of expensive reagents $<1 \mathrm{uL} \&$ increments of $0.1 \mathrm{uL}$

- Dilutions only go so far

- Wet \& dry dispense

- 1536 not going to work in the near term

- sequencers in $\mathbf{3 8 4}$ format

- no thermal cycling

- Physical constraints of laboratory space, big issue currently (next slide)
2006 Community

Sequencing Program

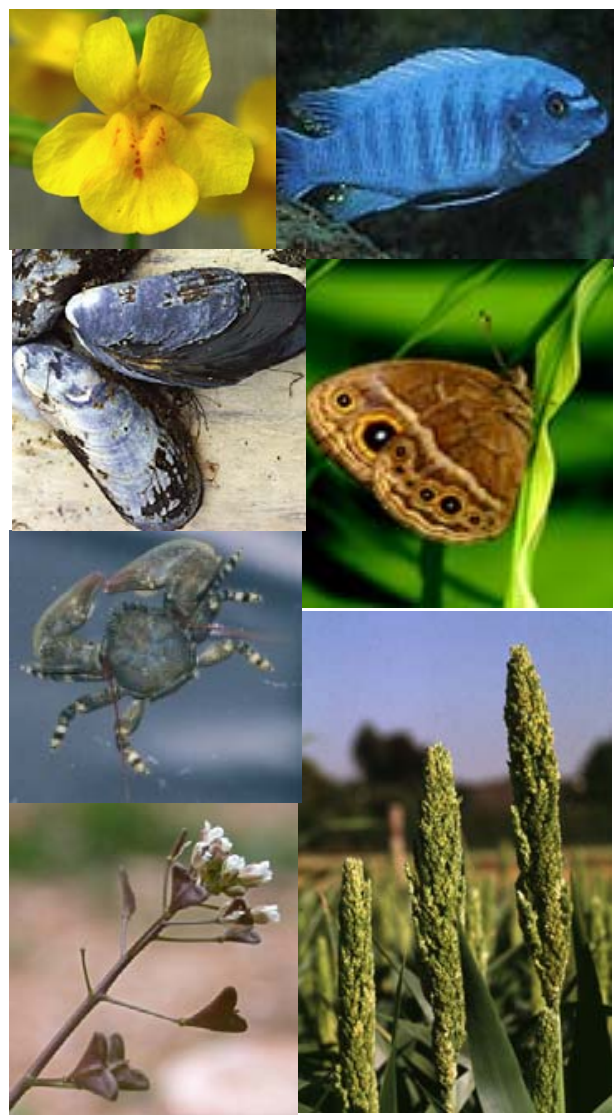




\section{JGI Facility Layout}

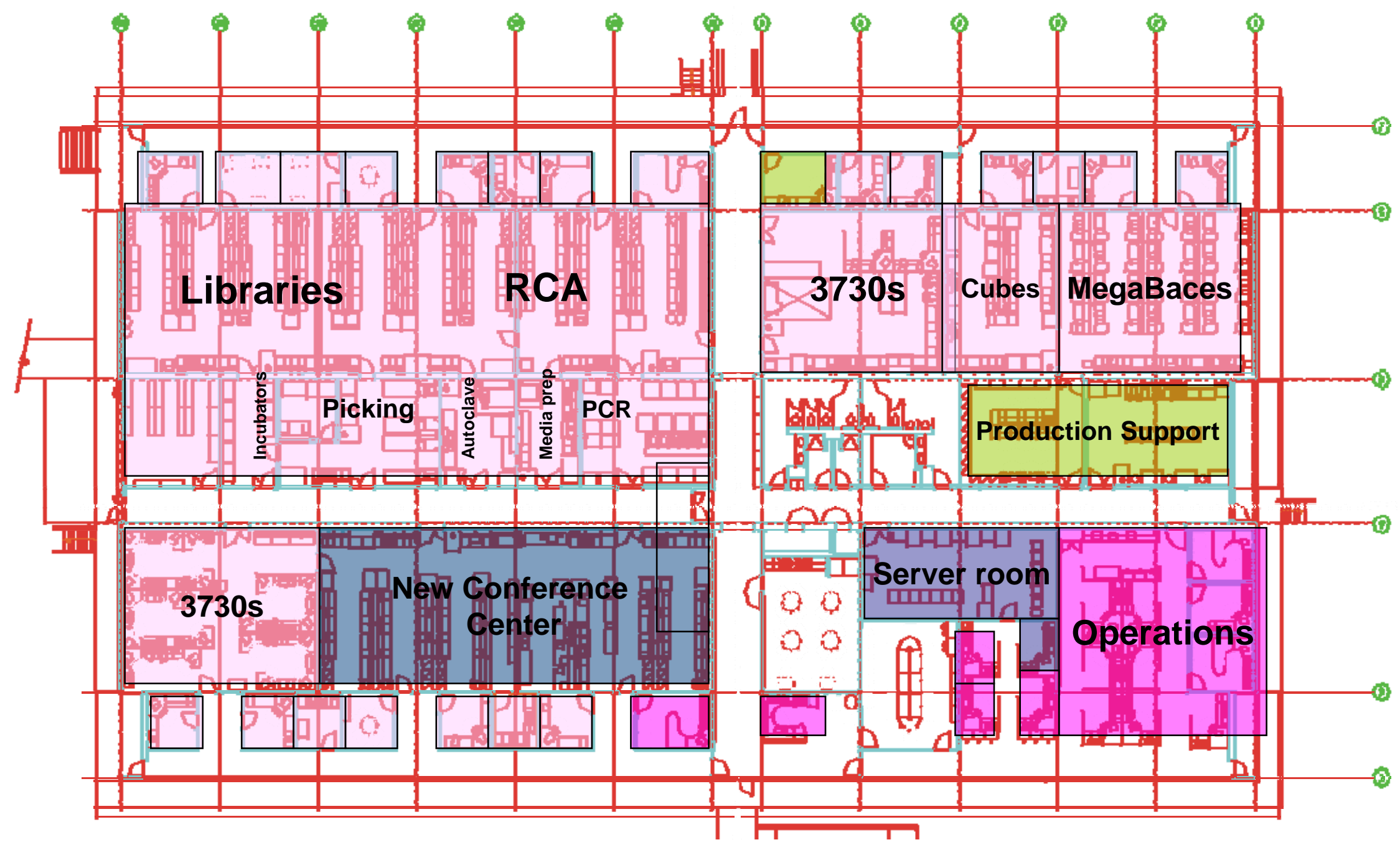




\section{Approach to Automation Selection}

- Performance

- Configuration, plate types (source \& destination, conditioned (thermal cycled \& incubated)), reagent types, throughput, software compatibility, GUI ease of use, barcode scanning, ancillary services

- Error Rate (defining major \& minor), how going to test stacker, pipettor, dispenser, barcode reader, tip wash.

- Measurement device, volumes, wet or dry dispense

- Safety - seismic restraint, ergonomics, hazards, "E" stop

- Integrated system test - method outline

- Operational testing

- Precision \%CV, accuracy, reliability, ergonomic design

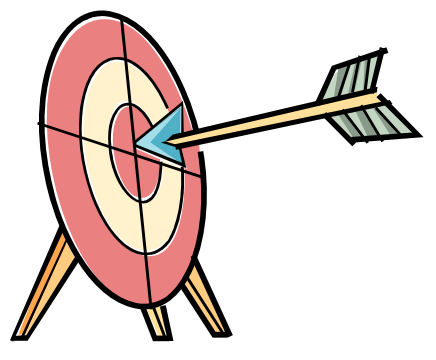




\section{Approach to Automation Selection}

- Cost

- Expense warranted, efficient use of tax $(4) \mathrm{y}$ payer money

- Delivery

- time, how, installation, training

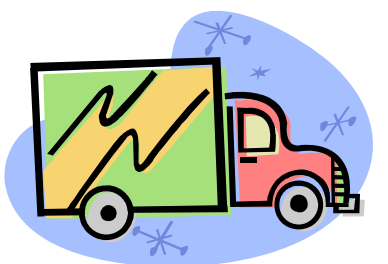

- Service

- field support, location of service engineers, response time, loaner parts,

की consumables supply 


\section{Approach to Automation Implementation}

- Technology Transfer

- New or upgrading instrument technology

- Internal process improvements

- New processes introduced to production from R\&D

- Performance

- Acceptance criteria

- Operational testing

- Duplicate partial batch

- Single production batch

- Statistical Analysis of Results

- Training

- SOP written during development phase, working draft

- Operator needs to know what to expect 


\section{summary}

- Brief history of the Joint Genome Institute

- DNA sequencing production line

- Increased throughput since 1999

- Instrumentation improvement highlights

- Our approach to successful selection \& implementation

\section{AGT C CGCGAAT ACAGGC T CGG T}

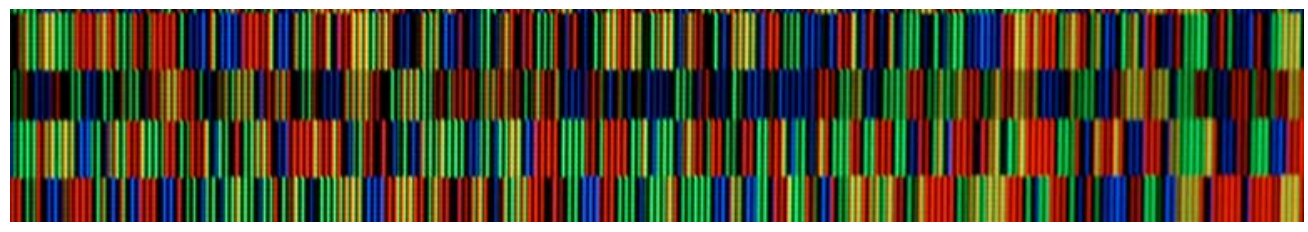




\section{More Information?}
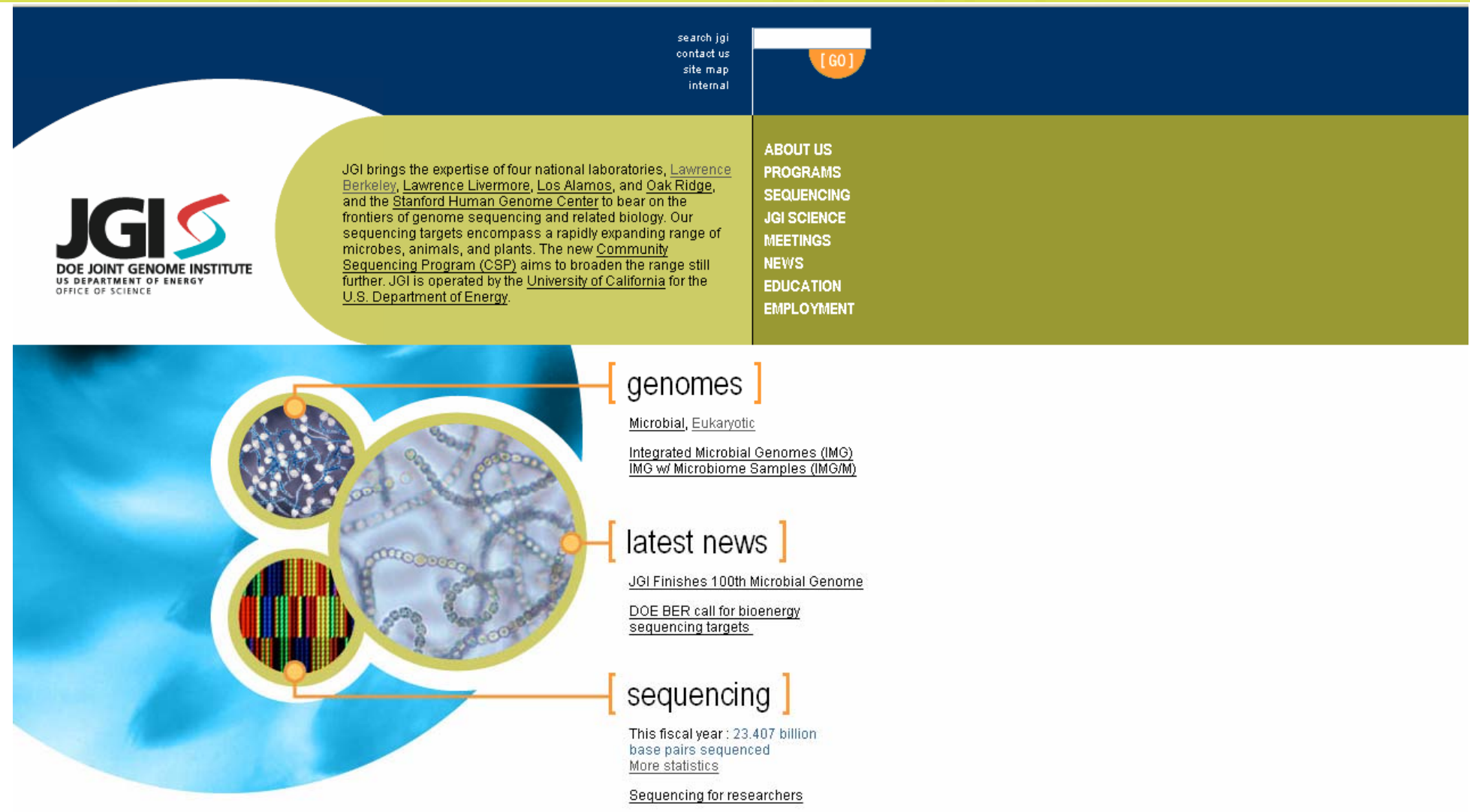

(iv) Co Office of (III) 
